\title{
Variations of moduli of parabolic bundles
}

\author{
Hans U. Boden, $\star$ Yi Hu \\ Department of Mathematics, University of Michigan, Ann Arbor, MI 48109, USA
}

Received: 11 August 1993/In revised form: 18 May 1994

Mathematics Subject Classification (1991): 14D20, 14H60, 14F32, 14F25

\section{Contents}

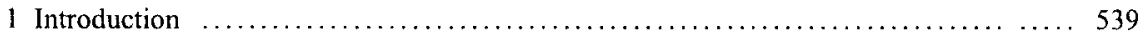

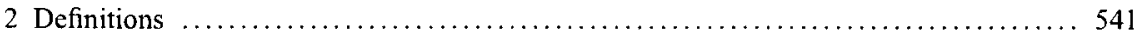

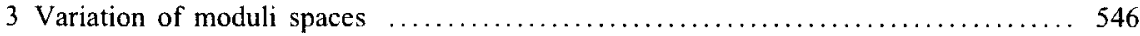

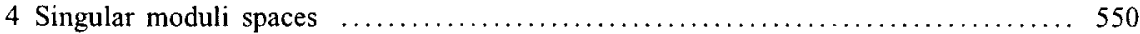

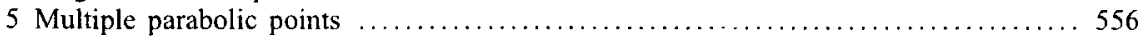

\section{Introduction}

In this paper, we study the moduli spaces of semistable parabolic bundles of arbitrary rank over a smooth curve $X$ with marked points in a finite subset $P$ of $X$. A parabolic bundle consists of a holomorphic bundle $\mathscr{E}$ over $X$ together with weighted flags in the fibers $\mathscr{E}_{p}$ for each $p \in P$. The moduli space $\mathscr{M}$ of semistable parabolic bundles was constructed by Mehta and Seshadri as the space of semistable holomorphic structures modulo $s$-equivalence. In [19], it is proved that $\mathscr{M}$ is a normal projective variety which is smooth for a generic choice of weights. They also observe that sufficiently close generic weights have isomorphic (in fact, identical) moduli.

We consider the problem further by studying the effect on the moduli of varying the weights. The space of admissible weights is a simplicial subset of $\mathbf{R}^{N}$ which we denote $W$. For $\alpha \in W$, we denote by $\mathscr{M}^{\alpha}$ the corresponding moduli space of parabolic bundles. The collection of weights with respect to which there exist strictly semistable bundles is a union of hyperplanes. Our main result is that if $\alpha$ and $\beta$ are generic weights which lie on either side of a given hyperplane $H$, then $\mathscr{M}^{\alpha}$ and $\mathscr{M}^{\beta}$ are related by a special birational transformation, 
which is similar to a flip in Mori theory. To see what this means, assume $\gamma \in H$ does not lie on any other hyperplane and let $\Sigma^{\prime \prime} \subset \mathscr{M}$ denote the set of $s$-equivalence classes of strictly semistable bundles. This is in general the singular locus of $\mathscr{M}$ with a few possible exceptions (cf. Remark 3.3). Then $\Sigma^{\prime}$ is smooth and the theorem states that there are two canonical projective maps $\phi_{\alpha}, \phi_{\beta}$

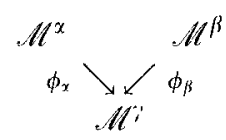

which are isomorphisms on the complement of $\Sigma^{\prime}$ and are $\mathbf{P}^{e_{x}}, \mathbf{P}^{e_{\beta}}$ (locally trivial) fibrations over $\Sigma^{\prime \prime}$. Moreover, $e_{x}+e_{\beta}+1=\operatorname{codim} \Sigma^{\prime}$.

This implies, for instance, the cohomology formula

$$
P\left(\mathscr{M}^{x}\right)=P\left(\mathscr{M}^{\beta}\right)+\left(P\left(\mathbf{P}^{e}\right)-P\left(\mathbf{P}^{e_{\sharp}}\right)\right) P\left(\Sigma^{\ddot{\gamma}}\right)
$$

where $P(Y)=\sum_{i} \operatorname{dim} \mathrm{H}^{i}(Y, \mathbf{Z}) t^{i}$ denotes the Poincare polynomial of $Y$. Additionally, one checks easily that one of the two maps $\phi_{x}$ and $\phi_{\beta}$ must be a small resolution (cf. [12]). In fact, we believe that for each singular moduli space $\mathscr{M}^{\prime}$, there is a smooth moduli space $\mathscr{M}^{x}$ so that the canonical map $\mathscr{M}^{x} \rightarrow \mathscr{M}^{\prime}$ is a small resolution (Conjecture 4.8)

Another related but much simpler problem is the effect of choosing $\gamma$ on the boundary of the weight space. This corresponds to the degeneration of the complete flag to a partial flag (see Proposition 3.4).

A natural next step is to understand the more singular moduli spaces. In Sect. 4, we use the Jordan-Hölder filtration of semistable bundles to introduce a natural stratification on the singular moduli $\mathscr{M}$ whose strata are products of moduli of lower rank and whose index set consists of $\gamma$-admissible partitions (Definition 4.3). (It is possible that this stratification coincides with the symplectic stratification of the representation variety of the fundamental group of surfaces given in [10] using the Mehta-Seshadri theorem.) We then prove a stratified version of Theorem 3.1 (see Theorem 4.5).

When the singular moduli is defined by a weight on the boundary of the weight space, things become a little different. As before, the Jordan-Hölder filtration defines a natural stratification on the moduli, except that now because the quotients of the filtration need not be distinct, the strata are symmetric products of lower dimensional moduli. Nevertheless, we prove a stratified version of Proposition 3.4 (see Proposition 4.7).

Although all of the results in Sect. 2 - Sect. 4 are proved under the assumption that the genus $g>1$ and that $X$ has one parabolic point, these assumptions are not essential. In the last section, we show how the results in the previous sections extend to these other cases. However, special care must be used for genus $g \leqq 1$, in particular we must assume that the top stratum (of stable bundles) is nonempty, which is not always the case.

For example, from Grothendieck's classification of holomorphic bundles over $\mathbf{P}^{1}$ [13], it follows that for certain (unfortunate) weights, the modult 
space is empty. In fact, it is not hard to see that for $g=0$ and any number of parabolic points, there exist generic weights whose moduli space is empty. Of course, there also exist generic weights with nonempty moduli. This change in moduli must occur when the weights are moved across some hyperplane which we call a "vanishing wall." Choosing weights on either side of a vanishing wall and analyzing the statement of the variation theorem in this context, we get an inductive proof of the rationality of the moduli in genus zero, confirming a conjecture in [5]. The key observation is that the moduli corresponding to a generic weight on the vanishing wall can contain only strictly semistable bundles and hence is a product of moduli of lower rank. This gives the induction.

It might be interesting to compare this work with that of some other people. Bradlow and Daskalopoulos have defined moduli of stable pairs [6], which are moduli parametrized by a number $t \in \mathbf{R}$. In the case of rank two, Thaddeus obtained results similar to Theorem 3.1 by varying $t$ [26]. His picture is like that of Guillemin-Sternberg for symplectic reductions of torus actions where crossing a wall results in a blowup followed by blowdown [15]. Our picture is more in the spirit of [11] and [17], where crossing a wall results in a "blowdown" followed by a "blowup." The first picture can be recovered from ours by considering the fibred product of the two canonical mornhisms of Theorem 3.1. From this point of view, the two maps from the fibred product to $\mathscr{M}^{\alpha}$ and $\mathscr{M}^{\beta}$ are blowdowns along $\phi_{\alpha}^{-1}\left(\Sigma^{\prime}\right)$ and $\phi_{\beta}^{-1}\left(\Sigma^{\prime}\right)$, respectively, and thus we see that the fibred product is the common blowup with exceptional divisor a $\left(\mathbf{P}^{e_{x}} \times \mathbf{P}^{e_{\beta}}\right)$-bundle over $\Sigma^{\prime}$. Recently, these results have been partially extended to GIT quotients of reductive algebraic group actions [8]

The paper is organized as follows. In all sections except Sect. 5, we assume that the genus of $X$ is larger than 1 to ensure that there are stable bundles for arbitrary weights. In Sect. 2, we give the definitions used throughout the paper. In Sect. 3, we state and prove the main results (Theorem 3.1 and Proposition 3.4). In Sect. 4, we extend the theory of the previous sections to very singular moduli. In Sect. 5, we consider the case of multiple parabolic points and prove that the moduli space of parabolic bundles is rational if $g=0$.

\section{Definitions}

\subsection{Parabolic bundles}

Suppose $X$ is a compact smooth complex curve with one marked point $p \in X$. Then a topological parabolic bundle over $X$ is a $\mathbf{C}^{n}$ bundle $E \stackrel{\pi}{\rightarrow} X$ with a weighted flag in the fiber over $p$, i.e.

$$
\begin{aligned}
& E_{p}=F_{1} \supset F_{2} \supset \ldots \supset F_{r} \supset 0, \\
& 0 \leqq \alpha_{1}<\alpha_{2}<\ldots<\alpha_{r}<1 .
\end{aligned}
$$

The flag is maximal in case $r=n$. Otherwise, we set $m_{\imath}=\operatorname{dim} F_{i} / F_{t+1}$, the multiplicity of $\alpha_{i}$. Define the parabolic degree of $E$ by 


$$
\operatorname{pardeg}_{x} E=\operatorname{deg} E+\sum_{i=1}^{r} m_{i} \alpha_{t},
$$

and the $\alpha$-slope of $E$ by

$$
\mu_{x}(E)=\frac{\operatorname{pardeg}_{x} E}{\operatorname{rank} E} .
$$

Remark 2.1. If $E^{\prime} \subset E$ is a subbundle with quotient $E^{\prime \prime}$, then both $E^{\prime}$ and $E^{\prime \prime}$ inherit the structure of a topological parabolic bundle from $E$ in a canonical way (for an explicit description, see the remark after Definition 1.8 of [19]).

By a slight abuse of notation, we will refer to the parabolic slope of a subbundle or quotient with the induced parabolic structure by $\mu_{x}\left(E^{\prime}\right)$ and $\mu_{\alpha}\left(E^{\prime \prime}\right)$.

The space of holomorphic structures on $E$, denoted $\mathscr{C}$, is an infinite dimensional affine space modeled on $\Omega^{0,1}($ End $E)$. The topological parabolic bundle $E$ together with a holomorphic structure $d \in \mathscr{C}$ is called a parabolic bundle and denoted $\mathscr{E}=(E, d)$.

Definition 2.2. An element $d \in \mathscr{C}$ or bundle $\mathscr{E}=(E, d)$ is $\alpha$-stable ( $\alpha$ semistable) if for any proper holomorphic subbundle $\mathscr{E}^{\prime} \subset \mathscr{E}$, we have $\mu_{x}\left(\mathscr{E}^{\prime}\right)<\mu_{x}(\mathscr{E})$ (respectively $\mu_{x}\left(\mathscr{E}^{\prime}\right) \leqq \mu_{x}(\mathscr{E})$ ). We denote by $\mathscr{C}_{s}^{\alpha}$ and $\mathscr{C}_{s s}^{\alpha}$ the spaces of $\alpha$-stable and $\alpha$-semistable bundles. Elements of the set $\mathscr{C}_{s s}^{\alpha}=\mathscr{C}_{s s}^{\alpha} \backslash \mathscr{C}_{s}^{\alpha}$ are called $\alpha$-strictly semistable bundles.

Now suppose that $\mathscr{E}_{v}$ is a parabolic bundle of rank $n_{v}$ for $v=1,2$, with parabolic structure over $p$ given by

$$
\begin{gathered}
\left(E_{v}\right)_{p}=F_{1}^{v} \supset F_{2}^{v} \supset \ldots \supset F_{r_{1}}^{v} \supset 0, \\
0 \leqq \alpha_{1}^{v}<\alpha_{2}^{v}<\ldots<\alpha_{r_{1}}^{y}<1,
\end{gathered}
$$

and multiplicities $m_{i}^{v}=\operatorname{dim} F_{i}^{v}-\operatorname{dim} F_{i+1}^{v}$. Suppose in what follows that $\{v, \eta\}=\{1,2\}$.

Definition 2.3. A map $\psi: \mathscr{E}_{v} \rightarrow \mathscr{E}_{\eta}$ of parabolic bundles is called a parabolic morphism if $\psi_{p}$ satisfies $\psi_{p}\left(F_{i}^{\prime}\right) \subseteq F_{j+1}^{\prime \prime}$ whenever $\alpha_{i}^{\prime}>\alpha_{j}^{\eta}$.

Denote by $\mathfrak{B a r} \mathfrak{J}_{0 m}\left(\mathscr{E}_{v}, \mathscr{E}_{\eta}\right)$ the sheaf of germs of parabolic morphisms from $\mathscr{E}_{v}$ to $\mathscr{E}_{\eta}$. This is clearly a subsheaf of $\operatorname{Som}\left(\mathscr{E}_{v}, \mathscr{E}_{\eta}\right)$. There are natural skyscraper sheaves $\mathscr{K}_{\text {in }}$ supported on the point $p \in X$ such that

$$
0 \rightarrow \operatorname{Par} \mathfrak{G o m}\left(\mathscr{E}_{v}, \mathscr{E}_{\eta}\right) \stackrel{i_{1}}{\longrightarrow} \operatorname{Som}\left(\mathscr{E}_{v}, \mathscr{E}_{\eta}\right) \longrightarrow \mathscr{K}_{v \eta} \rightarrow 0
$$

is a short exact sequence of sheaves. For any sheaf $W$, we use $\chi(W)$ to denote its Poincaré-Euler characteristic.

Lemma 2.4. Let $S_{e}$ be the set of pairs $(i, j)$ with $\alpha_{i}^{r}=\alpha_{j}^{\eta}$. Then

$$
\chi\left(\mathscr{K}_{i \eta}\right)+\chi\left(\mathscr{K}_{\eta}\right)=n_{\eta} n_{\eta}-\sum_{(i, j) \in S_{e}} m_{i}^{\prime \prime} m_{j}^{\eta}
$$


Proof.. Let $T_{v y}$ be the index set consisting of pairs $(i, j)$ with $1 \leqq i \leqq r_{\mathrm{r}}$ and $1 \leqq j \leqq r_{\eta}$. We will show directly that

$$
\chi\left(\mathscr{K}_{v \eta \eta}\right)=\sum_{(t, j) \in S_{v \eta}} m_{i}^{\prime \prime} m_{j}^{\eta},
$$

where $S_{v, j}=\left\{(i, j) \in T_{\eta \eta} \mid \alpha_{i}^{\eta}>\alpha_{j}^{\eta}\right\}$. Using transposition to identify $T_{i n}$ and $T_{\eta}$ (denoted $T$ ), we see that $S_{v \eta}$ and $S_{\eta v}$ are disjoint subsets of $T$ with complement $S_{e}$ and the lemma now follows from equation (2). Since $\chi\left(\mathscr{K}_{\text {vi }}\right)=\operatorname{dim} H^{0}\left(\mathscr{K}_{\text {vi }}\right)=\operatorname{dim}\left(\mathscr{K}_{i \eta}\right)_{p}$, to show equation (2), we just need to identify the cokernel of $i_{v \eta}$ on the stalks of (1) at $p$.

For this we introduce the following notation: $t$ is a local holomorphic coordinate on $X$ so that the parabolic point $p$ corresponds to $t=0 ;\left\{e_{1}^{\prime \prime}\right\}$ and $\left\{e_{j}^{n}\right\}$ are bases for $E_{p}^{v}$ and $E_{p}^{\prime \prime}$ so that $F_{h}^{r}$ is spanned by $\left\{e_{l}^{v} \mid m_{1}^{v}+\ldots+m_{h-1}^{v}<\right.$ $\left.i \leqq n_{v}\right\}$ and $F_{k}^{\eta}$ is spanned by $\left\{e_{j}^{\eta} \mid m_{1}^{\eta}+\ldots+m_{k-1}^{\eta}<j \leqq n_{\eta}\right\}$ for all $h$ and $k ;\left(a_{1}^{r}, \ldots, a_{n_{1}}^{v}\right)$ and $\left(a_{1}^{\eta}, \ldots, a_{n_{\eta}}^{\eta}\right)$ are the weights $\left(\alpha_{1}^{v}, \ldots, \alpha_{r_{1}}^{v}\right)$ and $\left(\alpha_{1}^{\eta}, \ldots, \alpha_{\eta_{\eta}}^{\eta}\right)$ repeated according to their multiplicities; and $\left\{x_{i j}^{\prime \prime \prime}\right\}$ is the set of coordinate functions on $n_{r} \times n_{\eta}$ matrices.

Using this notation, it follows that $\mathfrak{5 0 m}\left(\mathscr{E}_{v}, \mathscr{E}_{\eta}\right)_{p}=\mathbf{C}[[t]]\left[x_{i j}^{v i n}\right]$. Define

$$
\tau_{i j}^{v \eta}=\left\{\begin{array}{cl}
x_{i j}^{v \eta} & \text { if } a_{i}^{v} \leqq a_{j}^{\eta}, \\
t x_{i j}^{\eta \eta} & \text { if } a_{i}^{v}>a_{j}^{\eta} .
\end{array}\right.
$$

Then, with respect to the chosen bases, it follows from Definition 2.3 that

$$
\mathfrak{B a r} \mathfrak{H o m}\left(\mathscr{E}_{n}, \mathscr{E}_{\eta}\right)_{p}=\mathbf{C}[[t]]\left[\tau_{i j}^{v \prime \prime}\right]
$$

The map $i_{v \eta}$ on the stalks of (1) is just the natural inclusion with cokernel a vector space of dimension $\sum_{(i, j) \in S_{1 \eta}} m_{l}^{r} m_{j}^{\eta}$, proving equation (2) and completing the proof of the lemma.

Remark 2.5. It follows from this proof that as a function of the weights, $\chi\left(\mathscr{K}_{i \eta}\right)$ is a step function constant along those components with $\alpha_{l}^{p} \neq \alpha_{j}^{\prime \prime}$ for all $i, j$.

\subsection{The space of weights}

We are interested in how the moduli of stable bundles depends on the choice of weights $\alpha=\left(\alpha_{1}, \ldots, \alpha_{r}\right)$. For this, suppose $E$ is a topological parabolic bundle with $\mu_{x}(E)=0$. Let $-k=\operatorname{deg} E$, and $n=\operatorname{rank} E$. To define the set of admissible weights (i.e. the $\alpha$ with $\mu_{x}(E)=0$ ), it is convenient to write $\alpha=\left(\alpha_{1}, \ldots, \alpha_{n}\right)$, where each weight is repeated according to its multiplicity. We denote this set of admissible weights by $W_{k}$, of simply $W$ if the index is clear from context.

$$
W=\left\{\alpha \mid 0 \leqq \alpha_{1} \leqq \ldots \leqq \alpha_{n}<1 \text { with } \sum_{t=1}^{n} \alpha_{t}=k\right\}
$$

We denote by $\stackrel{\circ}{W}$ and $\partial W$ the interior and boundary of $W$, the latter of which we split into the two subsets: 


$$
\begin{aligned}
& \partial_{e} W=\left\{\alpha \in W \mid \alpha_{l}=\alpha_{i+1} \text { for some } 1 \leqq i<n\right\}, \\
& \partial_{0} W=\left\{\alpha \in W \mid \alpha_{1}=0 \text { and } \alpha_{i} \neq \alpha_{i+1} \quad \text { for all } 1 \leqq i<n\right\},
\end{aligned}
$$

because their moduli behave quite differently.

For generic weights, we have $\mathscr{C}_{s s s}^{\alpha}=\varnothing$. In fact, suppose $\alpha \in \stackrel{\circ}{W}$ has $\mathscr{C}_{s s s}^{x} \neq \varnothing$. Then there is a parabolic bundle $\mathscr{E}$ with proper subbundle $\mathscr{E}^{\prime}$ and $\mu_{x}\left(\mathscr{E}^{\prime}\right)=\mu_{x}(\mathscr{E})=0$. If $-k^{\prime}$ and $n^{\prime}$ are the degree and rank of $\mathscr{E}^{\prime}$, and $0 \leqq$ $\alpha_{\sigma(1)}<\ldots<\alpha_{\sigma\left(n^{\prime}\right)}<1$ are the weights of the natural parabolic structure induced on $\mathscr{E}^{\prime}$, then it follows that

$$
\sum_{i=1}^{n^{\prime}} \alpha_{\sigma(i)}=k^{\prime}
$$

Let $\xi=\left(\sigma, k^{\prime}, n^{\prime}\right)$ and define $H_{\xi} \subset W$ by

$$
H_{\zeta}=\left\{\alpha \in W \mid \sum_{i=1}^{n^{\prime}} \alpha_{\sigma(i)}=k^{\prime}\right\} .
$$

Then we have proved one direction of the following lemma.

Lemma 2.6. $\mathscr{C}_{s s s}^{x} \neq \varnothing \Leftrightarrow \alpha \in H_{\xi}$ for some $\xi$.

Proof. ( $\Leftarrow$ ) Use the construction for the direct sum of parabolic bundles ([24], p. 68).

Notice that $H_{\zeta}$ is a real hyperplane in $W$. We call the connected components (which are convex) of $W \backslash \bigcup_{\zeta} H_{\xi}$ top chambers. We also refer to the weights in top chambers as generic weights. Notice that for $\alpha \in W \backslash \bigcup_{\xi} H_{\xi}$ and for any proper subbundle $\mathscr{E}^{\prime}$ of $\mathscr{E}, \mu_{\alpha}\left(\mathscr{E}^{\prime}\right) \neq 0$. Thus, if $\alpha$ and $\beta$ are weights belonging to the same top chamber, then it is not hard to see that $\mathscr{C}_{s}^{x}=\mathscr{C}_{s}^{\beta}$. One can actually say more. We refer to connected components of

$$
\left(H_{\zeta_{1}} \cap \ldots \cap H_{\zeta_{n}}\right) \backslash\left(\bigcup_{\xi^{\prime} \neq \xi_{1}, \ldots, \xi_{n}} H_{\xi^{\prime}}\right)
$$

as chambers. These are the open convex faces of the top chambers.

Lemma 2.7. If $\alpha, \beta$ are in the same chamber, then $\mathscr{C}_{s}^{\alpha}=\mathscr{C}_{s}^{\beta}$ and $\mathscr{C}_{s s}^{\alpha}=\mathscr{C}_{s s}^{\beta}$.

Proof.. To prove this, we show set inclusions one way, and the reverse inclusions then follow from an identical argument. To start, let $\alpha_{t}=(1-t) \alpha+t \beta$ be the straight line from $\alpha$ to $\beta$, which is contained within the chamber by convexity. To see that $\mathscr{C}_{s s}^{x} \subseteq \mathscr{C}_{s s}^{\beta}$, suppose to the contrary some $d \in \mathscr{C}_{s s}^{x}$ is not an element of $\mathscr{C}_{s s}^{\beta}$. Then there is a holomorphic subbundle $\mathscr{E}^{\prime}$ of $\mathscr{E}$ with $\mu_{\beta}\left(\mathscr{E}^{\prime}\right)>0$. But certainly $\mu_{\alpha}\left(\mathscr{E}^{\prime}\right) \leqq 0$ by $\alpha$-semistability of $\mathscr{E}$. It then follows from the Intermediate Value Theorem that there is some $\alpha^{\prime}\left(=\alpha_{t_{0}}\right.$ for $\left.t_{0} \in[0,1)\right)$ with $\mu_{x^{\prime}}\left(\mathscr{E}^{\prime}\right)=0$. But this implies that $\alpha^{\prime} \in H_{\xi^{\prime}}$ for some hyperplane $H_{\xi^{\prime}}$ not containing $\beta$, a contradiction.

To see that $\mathscr{C}_{s}^{\alpha} \subseteq \mathscr{C}_{s}^{\beta}$, suppose $d \in \mathscr{C}_{s}^{x}$ is not an element of $\mathscr{C}_{s}^{\beta}$. Then $d \in \mathscr{C}_{s s s}^{\beta}$ and arguing as above, this implies that $\beta \in H_{\zeta^{\prime}}$ for some hyperplane $H_{\check{\zeta}^{\prime}}$ not containing $\alpha$, again a contradiction. 


\subsection{The moduli spaces}

We are ultimately interested in comparing different moduli of parabolic bundles. Suppose $\mathscr{E} \in \mathscr{C}_{s s}^{x}$. Then the Jordan-Hölder filtration of $\mathscr{E}$ is

$$
0 \subset \mathscr{E}_{1} \subset \mathscr{E}_{2} \subset \ldots \subset \mathscr{E}_{R}=\mathscr{E}
$$

where each quotient $D_{i}=\mathscr{E}_{i} / \mathscr{E}_{i-1}$ is $\alpha$-stable. (This terminology is justified by Remark 2.1.) This filtration is not canonical, but the associated graded bundle

$$
\mathbf{g r}_{x} \mathscr{E}=\bigoplus_{i=1}^{R} D_{i}
$$

is canonical. The moduli space $\mathscr{M}^{x}$, as a normal projective variety, is constructed by identifying $\mathscr{E}$ with $\mathscr{E}$ if $\mathbf{g r}_{x} \mathscr{E} \cong \mathbf{g r}_{x} \mathscr{E}$ as holomorphic parabolic bundles (see the definition preceding Theorem 4.1 of [19] and p. 178 of [20]). We call two holomorphic bundles $\mathscr{E}$ and $\mathscr{E}^{\prime}$ s-equivalent if $\mathbf{g r}_{x} \mathscr{E} \cong \mathbf{g r}_{x} \mathscr{E}^{\prime}$. When it is clear from context, we drop the subscript and write gr $\mathscr{E}$.

Theorem 2.8. (Theorem 4.1, [19]) If $\mathscr{C}_{s s s}^{x}=\varnothing$ and $\alpha \in \stackrel{\circ}{W}$, then $\mathscr{M}^{\alpha}$ is a smooth projective variety of dimension $(g-1) n^{2}+1+n(n-1) / 2$.

Although it is not necessary for the purpose of this paper, as an alternative point of view, we can describe the moduli spaces in terms of the gauge group $\mathscr{G}^{x}$. Suppose that $E$ is a topological parabolic bundle with weights $\alpha$, let $\mathscr{G}^{x}$ denote the group of bundle automorphism of $E$ preserving the parabolic structure:

$\mathscr{G}^{\alpha}=\left\{g: E \rightarrow E \mid \pi \circ g=\pi\right.$ and $g_{x} \in G L(n, \mathbf{C})$ for all $x \in X$ and $\left.g_{p}\left(F_{l}\right) \subseteq F_{l}\right\}$

Notice $\mathscr{G}^{x}$ depends only on the quasi-parabolic structure, i.e. on the unveighted flag structure. The gauge group acts on the space $\mathscr{C}$ of holomorphic bundles; if $g \in \mathscr{G}^{x}$ and $d \in \mathscr{C}$, then $g \cdot d=g \circ d \circ g^{-1}=d+g\left(d g^{-1}\right)$. The gauge orbit $\mathscr{G}^{x} \cdot \mathscr{E}$ is just the collection of parabolic bundles isomorphic to $\mathscr{E}$. Thus, two $\alpha$-stable bundles are $s$-equivalent if and only if they lie in the same gauge orbit. Although not entirely obvious, it is a consequence of [4] that two $\alpha$ semistable bundles are $s$-equivalent if and only if the closures of their gauge orbits have nontrivial intersection. Thus, we see $\mathscr{H}^{x}=\mathscr{C}_{s s}^{x} / \mathscr{G}^{x}$ where two orbits are identified if their closures in $\mathscr{C}_{s s}^{x}$ have nonempty intersection. Although we adopt a notation reminiscent of quotients in geometric invariant theory, it should be pointed out that $\mathscr{G}^{x}$ is not a reductive group.

Remark 2.9. Lemma 2.7 shows that if $\alpha$ and $\beta$ lie in the same chamber (top or otherwise), then the moduli $\mathscr{M}^{\alpha}$ and $\mathscr{M}^{\beta}$ are not only isomorphic, but actually identical. 


\section{Variation of moduli spaces}

\subsection{Statement of theorems}

We are now in a position to state the main result. Take $E$ a topological parabolic bundle and suppose $\alpha, \beta$ are weights in adjacent top chambers. Let $H_{\xi}$ be the hyperplane separating $\alpha$ and $\beta$ and choose $\gamma \in H_{\xi}$ so that $\gamma$ is not on any other hyperplane. Set $\Sigma^{r}$ to be $\mathscr{C}_{s s s}^{\prime}$ modulo $s$-equivalence. Notice that since $\gamma$ lies on only one hyperplane in $W, \Sigma^{\prime}$ is nonsingular. In fact, $\Sigma^{\prime \prime}$ is isomorphic to a product of lower dimensional moduli (see equation (5)).

Theorem 3.1. $\mathscr{M}^{\alpha}$ and $\mathscr{M}^{\beta}$ are related by a special birational transformation, that is, there are canonical projective maps

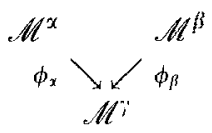

so that

(a) along $\mathscr{M}^{i} \backslash \Sigma^{i}, \phi_{x}$ and $\phi_{\beta}$ are isomorphisms,

(b) along $\Sigma^{\prime}, \phi_{\alpha}$ and $\phi_{\beta}$ are $\mathbf{P}^{e_{x}}$ and $\mathbf{P}^{e_{\beta}}$ fibrations, and

(c) $e_{x}+e_{\beta}+1=\operatorname{codim} \Sigma$ '.

Assume, without loss of generality, that $e_{x} \leqq e_{\beta}$. Then from part (c) of Theorem 3.1, we obtain that $\phi_{x}$ is a small resolution [12]. Note that if $f: X \rightarrow Y$ is small, then it induces an isomorphism between intersection homology groups of $X$ and $Y$ (loc. cit.).

Using the theorem, we can compare the cohomologies of $\mathscr{M}^{\alpha}$ and $\mathscr{M}^{\beta}$ to the intersection cohomology of $\mathscr{M}^{\prime}$. If $X$ is a smooth variety, we use $P(X)$ to denote its Poincaré polynomial, taken with rational coefficients. In case $X$ is singular, we use $I P(X)$ to denote the intersection Poincare polynomial and, for $x \in X, I P_{x}(X)$ for the local intersection Poincare polynomial, using middle perversity (see [12] for precise definitions).

Now we state a corollary of Theorem 3.1, whose proof follows from the general arguments given in Theorem 2.8 and Corollary 2.9 of [17].

Corollary 3.2. Let $\alpha, \beta$, and $\gamma$ be as in Theorem 3.1 and suppose $x \in \Sigma$. Then

(a) $P\left(\mathscr{M}^{x}\right)=I P\left(\mathscr{M}^{i}\right)+\left(P\left(\mathbf{P}^{e_{x}}\right)-I P_{x}\left(\mathscr{M}^{\prime}\right)\right) P\left(\Sigma^{i}\right)$,

(b) $P\left(\mathscr{M}^{\beta}\right)=I P\left(\mathscr{M}^{i}\right)+\left(P\left(\mathbf{P}^{e_{\beta}}\right)-I P_{x}\left(\mathscr{M}^{i}\right)\right) P\left(\Sigma^{i}\right)$,

(c) $I P_{x}\left(\mathscr{M}^{i}\right)= \begin{cases}P\left(\mathbf{P}^{e_{x}}\right) & \text { if } e_{x} \leqq e_{\beta}, \\ P\left(\mathbf{P}^{e_{j}}\right) & \text { if } e_{\beta} \leqq e_{x} .\end{cases}$

Remark 3.3. If $e_{x} \neq 0 \neq e_{\beta}$, then by the above corollary, $I P_{x}\left(\mathscr{M}^{i}\right) \neq 1$ for every $x \in \Sigma^{\prime}$. That is, $\Sigma^{i}$ consists of singular points. Since the complement of $\Sigma^{\prime}$ is smooth, we found that $\Sigma^{\prime \prime}$ is precisely the singular locus of $\mathscr{M}^{\prime}$. If one of $e_{x}$ and $e_{\beta}$ is zero, which occurs only when $g \leqq 1$, then the corresponding morphism is an isomorphism because the moduli spaces are all normal varieties. 
It is observed in Proposition 5.1 of [5] that if $\mathscr{C}_{s s s}^{x}=\varnothing$, then $\mathscr{A}^{x}$ is torsion free. Combined with the above corollary, this implies that

$$
P\left(\mathscr{M}^{\alpha}\right)=P\left(\mathscr{M}^{\beta}\right)+\left(P\left(\mathbf{P}^{e_{x}}\right)-P\left(\mathbf{P}^{e_{\beta}}\right)\right) P\left(\Sigma^{\prime}\right),
$$

on the level of integral cohomology.

The next proposition describes the map between moduli induced by a degeneration of flag structure. Suppose that $\delta \in \partial_{e} W$ and $\alpha \in \stackrel{\circ}{W}$ are in the closure of the same chamber and do not lie on any hyperplanes.

Proposition 3.4. There is natural projection $\mathscr{M}^{x} \rightarrow \mathscr{M}^{\delta}$ that is a fibration with fiber a product of flag varieties.

If instead $\delta$ were chosen in the other component of the boundary, $\partial_{0} W$, then because the quasi-parabolic structures of $\alpha$ and $\delta$ in this case are identical, the map between the moduli spaces would not be a fibration. This map should really be thought of in the context of the variation theorem (for one-sided hyperplanes). Just as in Theorem 3.1, there is a special birational transformation $\phi_{x}$ : $\mathscr{M}^{\alpha} \rightarrow \mathscr{A}^{\delta}$ which is an isomorphism along $\mathscr{M}^{i} \backslash \Sigma i$ and which is a $\mathbf{P}^{e^{\alpha}}$ bundle along $\Sigma^{\prime}$.

\subsection{Proofs}

Proof of theorem 3.1. We first show how to construct the map $\phi_{\alpha}$ and then prove it has the correct properties. The same results for $\phi_{\beta}$ follow by an identical argument.

Lemma 3.5. There are natural inclusions: (a) $\mathscr{C}_{s s}^{x} \subset \mathscr{C}_{s s}^{\prime \prime}$, and (b) $\mathscr{C}_{s}^{i} \subset \mathscr{C}_{s}^{x}$.

Proof. (a) Let $\alpha_{t}$ be the straight line $\alpha_{t}=(1-t) \alpha+t \gamma$. It follows that $\mathscr{C}_{s s s}^{x_{1}}=\varnothing$ for $0 \leqq t<1$. Suppose $\mathscr{E} \in \mathscr{C}_{s s}^{x}=\mathscr{C}_{s}^{x}$ and $\mathscr{E} \notin \mathscr{C}_{s s}^{\prime}$. Then we have a proper subbundle $\mathscr{E}^{\prime}$ of $\mathscr{E}$ with $\mu_{i}\left(\mathscr{E}^{\prime}\right)>0$. Since $\mathscr{E}$ is $\alpha$-stable, $\mu_{x}\left(\mathscr{E}^{\prime}\right)<0$. But $\mu_{x_{t}}\left(\mathscr{E}^{\prime}\right)$ varies continuously with $t \in[0,1]$. The Intermediate Value Theorem implies that there is a $t_{0} \in(0,1)$ so that $\mu_{\alpha^{\prime}}\left(\mathscr{E}^{\prime \prime}\right)=0$ where $\alpha^{\prime}=\alpha_{t_{0}}$. But this implies that $\alpha^{\prime} \in H_{\xi}$ for some $\xi$, a contradiction.

(b) Now suppose $\mathscr{E} \in \mathscr{C}_{s}^{\prime}$ and that there is a subbundle $\mathscr{E}^{\prime}$ of $\mathscr{E}$ so that $\mu_{x}\left(\mathscr{E}^{\prime}\right)>0$. By $\gamma$-stability of $\mathscr{E}, \mu_{;}\left(\mathscr{E}^{\prime}\right)<0$. Thus, by the IVT, there is a $t_{0} \in(0,1)$ so that setting $\alpha^{\prime}=\alpha_{t_{0}}$, we have $\mu_{\alpha^{\prime}}\left(\delta^{\prime \prime}\right)=0$, again a contradiction.

Lemma 3.6. The map $\phi_{x}: \mathscr{H}^{\alpha} \rightarrow \mathscr{H}^{i}$ induced by inclusion $\mathscr{C}_{s}^{\alpha} \subset \mathscr{C}_{s s}^{i}$ is a projective birational map which is an isomorphism along $\mathscr{H} \backslash \backslash \Sigma^{\prime}$.

Proof. We claim that the inclusion $\mathscr{C}_{s}^{x} \subset \mathscr{C}_{s s}^{\prime}$ descends to a map $\phi_{x}$ after taking $s$-equivalence. To see this we can appeal to the proof of Theorem 4.1 of [19] (pp. 225-235) to translate the problem to geometric invariant theory where the property readily follows. To avoid a preponderance of notation we adopt that of [19] and supply brief explanations where necessary. Using the Hilbert Scheme of coherent sheaves over $X$, Mehta and Seshadri construct a smooth variety $R$ and a flag bundle $\tilde{R}$ over $R$. Let $\tilde{R}_{s s}^{\prime}\left(\tilde{R}_{s}^{\prime}\right)$ denote the set of 
points representing $\gamma$-semistable (stable) parabolic bundles. There is a smooth projective variety $Z$ which is a product of Grassmannians of certain dimensional quotients of the trivial bundle $\mathcal{C}_{X}^{\oplus N}$ of rank $N$ over $X$ for some integer $N$ (loc. cit, pp. 226). The weight $\gamma$ induces a polarization on $Z$ which gives rise to a linear action of the group $G=S L\left(\mathcal{C}_{X}^{\oplus N}\right)$ on $Z$. Let $Z_{s s}^{i}\left(Z_{s}^{*}\right)$ be the set of semistable (stable) points with respect to the linear action of $G$ induced by $\gamma$ in the sense of geometric invariant theory. There is a map $T: \tilde{R} \rightarrow$ $Z$ such that $T$ respects stability (cf. Proposition 4.2 of [19]). In particular, $T\left(\tilde{R}_{s s}^{\prime}\right) \subset Z_{s s}^{\prime \prime}$ and $T\left(\tilde{R}_{s}^{\prime}\right) \subset Z_{s}^{\prime}$. Let $M^{\prime}$ be the image of $\tilde{R}_{s s}^{\prime}$ in $Z_{s s}^{\prime} / / G$ and $\tilde{M}^{\prime}$ be its normalization. Then $\mathscr{M}^{i}$ as a normal variety is constructed as $\tilde{M}^{\prime}$ (this follows from Proposition 4.4 of [19]). Similarly let $\tilde{R}_{s s}^{\alpha}\left(\tilde{R}_{s}^{x}\right)$ denote the set of points representing $\alpha$-semistable (stable) parabolic bundles. Then $\tilde{R}_{s s}^{\alpha} \subset \tilde{R}_{s s}^{\prime \prime}$ and $\tilde{R}_{s}^{\alpha} \supset \tilde{R}_{s}^{\gamma}$. The moduli $\mathscr{M}^{\alpha}$ is constructed similarly by using the linear action of $G$ on $Z$ induced by the weight $\alpha$. However we have $T\left(\tilde{R}_{s s}^{x}\right) \subset T\left(\tilde{R}_{s s}^{\prime}\right)$ and $T\left(\tilde{R}_{s}^{x}\right) \supset T\left(\tilde{R}_{s}^{i}\right)$. Hence it readily follows from geometric invariant theory that the above inclusions induce an algebraic map from $M^{\alpha}=T\left(\tilde{R}_{s s}^{\alpha}\right) / / G(\subset$ $\left.Z_{s s}^{\alpha} / / G\right)$ to $M^{i}=T\left(\tilde{R}_{s s}^{i}\right) / / G\left(\subset Z_{s s}^{\prime \prime} / / G\right)$. Since $M^{x} \rightarrow M^{i}$ is obviously a birational morphism, it follows that it can be uniquely lifted to their normalizations $\phi_{\alpha}$ : $\tilde{M}^{\alpha} \rightarrow \tilde{M}^{i}$, and this is the morphism we desire. Clearly $T\left(\tilde{R}_{s}^{\alpha}\right) \supset T\left(\tilde{R}_{s}^{\prime}\right)$ implies that $\phi_{x}$ is an isomorphism on $\mathscr{M}^{i} \backslash \Sigma^{i}$ which is the normalization of $T\left(\tilde{R}_{s}^{i}\right) / G$. The projectivity of the morphism $\phi_{x}$ follows from the fact that any algebraic map between projective varieties is projective.

With the next lemma, we show how to identify $\phi_{\alpha}^{-1}\left(\left[\mathscr{E}_{1}\right]\right)$ for $\mathscr{E}_{1} \in \mathscr{C}_{s s s}^{\prime}$ with certain nontrivial extensions, where $\left[\mathscr{E}_{1}\right]$ denotes the set of $\gamma$-semistable bundles $s$-equivalent to $\mathscr{E}_{1}$. Since $\gamma \in H_{\xi}$ lies on a unique hyperplane, $\mathscr{E}_{1} \in \mathscr{C}_{s s s}^{\prime}$ implies $\operatorname{gr}_{i} \mathscr{E}_{1}=S \oplus Q$ for some $\gamma$-stable bundles $S$ and $Q$. By the definition of $s$-equivalence, it follows that a $\gamma$-semistable bundle $\mathscr{E}$ is $s$-equivalent to $\mathscr{E}_{1}$ if and only if $\operatorname{gr}_{-} \mathscr{E}=S \oplus Q$, i.e. if $E$ is a parabolic extension of $S$ by $Q$ or of $Q$ by $S$. Note that we are using the parabolic structure on $\mathscr{E}_{1}$ to give parabolic structures to $S$ and $Q$ as subbundle and quotient, and then assembling these to give a parabolic structure to $\mathscr{E}$.

Now consider what happens when the weights are varied. Then $\mathscr{E}_{1}$ can be considered as a parabolic bundle with weight $\alpha$, and while it may not be $\alpha$ stable, we can identify which extensions in $\left[\mathscr{E}_{1}\right]$ will be $\alpha$-stable. Of course, we need to know which of the bundles $S$ and $Q$ has positive $\alpha$-slope. (Note that $S$ and $Q$ are now thought of as bundles whose parabolic structure is determined from the $\alpha$-parabolic structure on $\mathscr{E}_{1}$.) Recall that $\operatorname{Par}_{\mathfrak{H o m}}(Q, S)$ denotes the sheaf of germs of parabolic morphisms from $Q$ to $S$.

Lemma 3.7. Suppose $\mathscr{E}_{1} \in \mathscr{C}_{s s s}^{\prime}$ with $\operatorname{gr} \mathscr{E}_{1}=S \oplus Q$ with $\mu_{x}(S)<0$ and $\mu_{x}(Q)>0$. Then

$$
\phi_{x}^{-1}\left(\left[\mathscr{E}_{1}\right]\right)=\operatorname{Proj}\left(\mathrm{H}^{1}\left(\operatorname{Parhom}_{\alpha}(Q, S)\right)\right) .
$$

Proof.. Suppose $\mathscr{E} \in \phi_{x}^{-1}\left(\left[\mathscr{E}_{1}\right]\right)$. Then $\operatorname{gr}_{.} \mathscr{E}=S \oplus Q$ which, since $\mathscr{E}$ is $\alpha$-stabie, gives the short exact sequence of parabolic bundles 


$$
0 \rightarrow S \rightarrow \mathscr{E} \rightarrow Q \rightarrow 0
$$

Again by $\alpha$-stability, $\mathscr{E}$ must be a nontrivial extension of $Q$ by $S$. Notice that because $\mu_{x}(Q)>0>\mu_{x}(S), \mathrm{H}^{0}\left(\mathfrak{P a r h}_{\mathfrak{y} 0 m_{x}}(Q, S)\right)=0$ (see Proposition 9 of [24]). Thus by standard homological algebra the nontrivial extension (4) is classified by a 1-dimensional linear subspace of $\operatorname{ParExt}_{x}^{1}(Q, S)$. However $\operatorname{Parhom}_{x}(Q, S)$ is a subsheaf of $\mathfrak{H o m m}_{x}(Q, S)$ and thus is locally free, and it follows that $\operatorname{ParExt}_{x}^{1}(Q, S)=\mathrm{H}^{1}\left(\operatorname{Par}_{\mathfrak{S O m}_{x}}(Q, S)\right)$. This shows inclusion one way, and the other inclusion follows from the claim that any bundle $\mathscr{E}$ which is given by a nontrivial extension of $Q$ by $S$ (as $\alpha$-parabolic bundles) is in fact $\alpha$-stable and thus is in $\phi_{\alpha}^{-1}\left(\left[\mathscr{E}_{1}\right]\right)$. For suppose to the contrary that $\mathscr{E}$ is not $\alpha$-stable. Then there is a proper subbundle $\mathscr{E}^{\prime}$ of $\mathscr{E}$ with $\mu_{x}\left(\mathscr{E}^{\prime}\right)>0$. But $\mu_{\gamma}\left(\mathscr{E}^{\prime \prime}\right)=0$ by $\gamma$-semistability of $\mathscr{E}$. Thus there is a short exact sequence $\mathscr{E}^{\prime} \rightarrow \mathscr{E} \rightarrow \mathscr{E}^{\prime \prime}$ so that gr $_{i} \mathscr{E}=\mathscr{E}^{\prime} \oplus \mathscr{E}^{\prime \prime}$. By its construction, gr. $_{i} \mathscr{E}=S \oplus Q$. So $\mathscr{E}^{\prime}$ is isomorphic to either $S$ or $Q$. Since the original extension was nontrivial, it follows that $\mathscr{E}^{\prime} \cong S$. But then $\mu_{x}(S)>0$, a contradiction.

This lemma also proves that $\phi_{\beta}^{-1}\left(\left[\mathscr{E}_{1}\right]\right)=\operatorname{Proj}\left(\mathrm{H}^{1}\left(\mathfrak{B a r} \mathfrak{H o m}_{\beta}(S, Q)\right)\right)$. Thus, $e_{x}=\mathrm{h}^{1}\left(\mathfrak{B a r}_{\mathfrak{S o m}}(Q, S)\right)-1$ and $e_{\beta}=\mathrm{h}^{1}\left(\mathfrak{B a r} \mathfrak{g o m} \mathrm{m}_{\beta}(S, Q)\right)-1$. We now compute $e_{x}$ and $e_{\beta}$ and show that $e_{x}+e_{\beta}+1=\operatorname{codim} \Sigma^{\prime}$.

Suppose that $\gamma$ lies on the hyperplane $H_{\xi}$ determined by $\xi=\left(\sigma . k_{1}, n_{1}\right)$. Let $k_{2}, n_{2}$ be the complementary degree and rank, so that any $\mathscr{E} \in \mathscr{C}_{s s s}^{i}$ has gr $\mathscr{E}=S \oplus Q$ where $\operatorname{rank} S=n_{1}, \operatorname{rank} Q=n_{2}$ and $\operatorname{deg} S=-k_{1}, \operatorname{deg} Q=-k_{2}$. By identifying $\Sigma^{\prime \prime}$ with the product of the moduli of stable parabolic bundles on $S$ and $Q$ with weights $\gamma^{\prime}, \gamma^{\prime \prime}$ where

$$
\gamma^{\prime}=\left(\gamma_{\sigma(1)}, \ldots, \gamma_{\sigma\left(n_{1}\right)}\right), \quad \gamma^{\prime \prime}=\left(\gamma_{\tau(1)}, \ldots, \gamma_{\tau\left(n_{2}\right)}\right),
$$

and $\sigma, \tau$ are complementary choice functions, it follows that

$$
\Sigma \Sigma^{\prime}=\mathscr{M}^{\prime} \times \mathscr{M}^{\prime \prime} \text {. }
$$

In particular, applying the dimension formula of Theorem 2.8 shows

$$
\operatorname{codim} \Sigma^{\prime}=n_{1} n_{2}(2 g-1)-1 .
$$

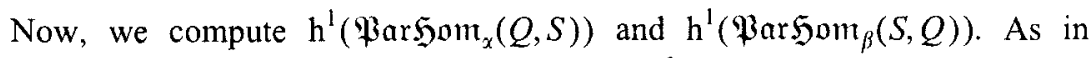
the proof of the previous lemma, we have $h^{0}\left(\mathfrak{B a r} \mathfrak{y}^{\circ} m_{x}(Q, S)\right)=0$ and

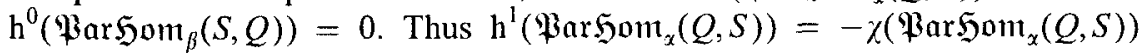
and $h^{1}\left(\mathfrak{P a r} \mathfrak{S o m}_{\beta}(S, Q)\right)=-\chi\left(\mathfrak{B a r} \mathfrak{j} \cup m_{\beta}(S, Q)\right)$. Using the short exact sequences of sheaves

$$
\begin{aligned}
& \mathfrak{B a r} \mathfrak{H} m_{\alpha}(Q, S) \rightarrow \mathfrak{H o m}(Q, S) \rightarrow \mathscr{H}, \\
& \mathfrak{B a r} 5 \mathrm{gom}_{\beta}(S, Q) \rightarrow \operatorname{5om}(S, Q) \rightarrow \mathscr{K},
\end{aligned}
$$

where $\mathscr{H}$ and $\mathscr{K}$ are skyscraper sheaves, it follows that

$$
\begin{aligned}
& \chi(\mathfrak{H a r h o m},(Q, S))=\chi(\mathfrak{H o m}(Q, S))-\chi(\mathscr{H}), \\
& \chi\left(\mathfrak{P a r} \mathfrak{g} \mathbf{m}_{\beta}(S, Q)\right)=\chi(\mathfrak{G o m}(S, Q))-\chi(\mathscr{K}) .
\end{aligned}
$$


The Weil formula ([16], p. 156) gives

$$
\begin{aligned}
& \chi(\mathfrak{S} \circ \mathrm{m}(Q, S))=n_{1} k_{2}-n_{2} k_{1}+n_{1} n_{2}(1-g), \\
& \chi(\mathfrak{S} \circ \mathrm{m}(S, Q))=n_{2} k_{1}-n_{1} k_{2}+n_{1} n_{2}(1-g) .
\end{aligned}
$$

Thus,

$$
\begin{aligned}
e_{x} & =\mathrm{h}^{1}\left(\mathfrak{B a r} \mathfrak{H o m}_{x}(Q, S)\right)-1 \\
& =-\chi\left(\mathfrak{B a r} \mathfrak{G o m}_{\alpha}(Q, S)\right)-1 \\
& =-\chi(\mathfrak{G o m}(Q, S)+\chi(\mathscr{H})-1 \\
& =n_{2} k_{1}-n_{1} k_{2}-n_{1} n_{2}(1-g)+\chi(\mathscr{H})-1
\end{aligned}
$$

and

$$
e_{\beta}=n_{1} k_{2}-n_{2} k_{1}-n_{1} n_{2}(1-g)+\chi(\mathscr{K})-1
$$

Thus

$$
e_{\alpha}+e_{\beta}+1=-2 n_{1} n_{2}(1-g)+n_{1} n_{2}-1=n_{1} n_{2}(2 g-1)-1
$$

since $\chi(\mathscr{H})+\chi(\mathscr{K})=n_{1} n_{2}$ by Lemma 2.4 (the weights of $S$ and $Q$ are necessarily distinct). This completes the proof of Theorem 3.1.

Remark 3.8. The projective fibrations identified in Theorem 3.1 are actually projectivizations of vector bundles over $\Sigma ;$ which are locally trivial in the Zariski topology. Construct the family $\mathscr{U}$ of parabolic vector bundles parameterized by $\Sigma^{\prime}$ as follows. Consider the two universal families parameterized by $\mathscr{M} i^{\prime}$ and $\mathscr{M} i^{\prime \prime}$ (Theorem 32 of [24]), pull them back to $\Sigma^{\prime} \times X$, and take the tensor product of the first with with the dual of the second and this defines the family $\mathscr{U}$. We have shown above that $\mathrm{H}^{0}\left(\mathscr{U}_{[E]}\right)=0$, and now it follows from Proposition 7.8.4 of [14] that the first direct image of $\mathscr{U}$ under the projection $\Sigma^{\prime} \times X \rightarrow \Sigma^{\prime}$ is locally free.

proof of Proposition 3.4. Given $\delta \in \hat{\partial}_{e} W$, choose $\alpha$ as in the proposition. Lemma 2.7 shows that $\mathscr{C}_{s s}^{x}=\mathscr{C}_{s}^{\chi}=\mathscr{C}_{s}^{\delta}=\mathscr{C}_{s s}^{\delta}$. Thus the projection $\mathscr{M}^{\chi} \rightarrow \mathscr{M}^{\delta}$ is simply the forgetful map which forgets that part of the flag that appears in the quasi-parabolic structure that defines $\alpha$ but does not occur in in the quasiparabolic structure that defines $\delta$. The proposition follows immediately. In fact, via the gauge group construction, the fiber is $\mathscr{G}^{\delta} / \mathscr{G}^{x}$, which is a product of flag varieties.

\section{Singular Moduli Spaces}

So far we have considered only moduli $\mathscr{M}$ where $\gamma$ is a general point in a hyperplane of the weight space. In this case, the set $\Sigma^{\prime \prime}$ is a nonsingular variety and so $\mathscr{M}$ ' has two strata

$$
\mathscr{M}^{\prime}=\left(\mathscr{M}^{\prime}-\Sigma^{\prime \prime}\right) \cup \Sigma^{\prime}
$$


One should expect more complicated singularities for $\mathscr{M}$ when $\gamma$ lies on an intersection of hyperplanes. Fortunately, the Jordan-Hölder filtration gives a natural stratification of $\mathscr{M} i$ which one can use to prove stratified versions of Theorem 3.1 and Proposition 3.4. We begin with a description of this stratification and its companion index set.

To start, we define a partial ordering on the set of weights by the face relations determined by the hyperplanes $H_{\zeta}$. Specifically, suppose that $\beta$ and $\gamma$ are weights contained in the chambers $F$ and $G$ respectively, then $\beta>\gamma$ in case $G$ is a proper face of $F$.

Proposition 4.1. If $\beta, \gamma \in \stackrel{\circ}{W}$ and $\beta>\gamma$, then the inclusion $\mathscr{C}_{s s}^{\beta} \subset \mathscr{C}_{s s}^{\ddot{\prime}}$ induces $a$ birational projective algebraic map $\phi_{\beta}: \mathscr{M}^{\beta} \rightarrow \mathscr{M}$ which is an isomorphism along $\mathscr{M}^{i} \backslash \Sigma^{\prime}$.

Proof.. An argument like that used to prove Lemma 3.5 shows that $\mathscr{C}_{s s}^{\beta} \subset \mathscr{C}_{s s}^{\ddot{\prime}}$ and $\mathscr{C}_{s}^{i} \subset \mathscr{C}_{s}^{\beta}$. The rest follows as in the proof of Theorem 3.1.

Remark 4.2. When $\mathscr{M}^{x}$ is nonsingular, $\phi_{x}$ is a resolution of singularities.

We now describe the stratification on $\mathscr{M}$ i obtained from the Jordan-Hölder filtration (3). The index set for this stratification is the finite set of all partitions of $\gamma=\left(\gamma_{1}, \ldots, \gamma_{n}\right)$ into sets

$$
\left(\gamma_{\sigma_{1}(1)}, \ldots, \gamma_{\sigma_{1}\left(n_{1}\right)}\right), \ldots,\left(\gamma_{\sigma_{R}(1)}, \ldots, \gamma_{\sigma_{R}\left(n_{R}\right)}\right)
$$

such that

$$
\sum_{j=1}^{n_{t}} \gamma_{\sigma_{l}(j)}=k_{l} \in \mathbf{Z}
$$

We call this a $\gamma$-partition and denote it with the letter $\xi$. Notice that $\xi$ is determined by three related partitions, $(\sigma, \mathbf{k}, \mathbf{n})$ where

$$
\begin{aligned}
& \sigma=\left(\sigma_{1}, \ldots, \sigma_{R}\right) \text { is a partition of the set }\{1, \ldots, n\}, \\
& \mathbf{k}=\left(k_{1}, \ldots, k_{R}\right) \text { is a partition of the integer } k, \\
& \mathbf{n}=\left(n_{1}, \ldots, n_{R}\right) \text { is the partition of the integer } n \text { determined by } \sigma .
\end{aligned}
$$

If $\gamma$ is an interior weight, then in fact $\xi$ is uniquely determined by $(\sigma, \mathbf{k}, \mathbf{n})$. But when $\gamma \in \partial_{e} W$, the representation of $\xi$ as $(\sigma, \mathbf{k}, \mathbf{n})$ may not be unique. This is clear by considering the effect of interchanging two equal weights (now repeated according to their multiplicity); although it will not change the $\gamma$ partition $\xi$, it may change $\sigma$. We will come back to this point when we discuss the stratified degeneration theorem. For now, we assume that $\gamma$ is an interior weight, so we can unambiguously write $\xi=(\sigma, \mathbf{k}, \mathbf{n})$.

We set $|\xi|=R$, the length of the partition. Notice that $\gamma$ admits a length 2 partition $\xi$ if and only if $\gamma$ lies on the hyperplane $H_{\xi}$.

Definition 4.3. For $\gamma \in W$, let $\pi(\gamma)=\{\xi \mid \xi$ is a $\gamma$-partition $\}$. 
If $\xi=(\sigma, \mathbf{k}, \mathbf{n})$ is a $\gamma$-partition, then both $\mathbf{k}$ and $\mathbf{n}$ are determined from $\sigma$.

Consider a strictly semistable bundle $\mathscr{E} \in \mathscr{C}_{s s s}^{\prime \prime}$ with gr $\mathscr{E}=D_{1} \oplus \ldots \oplus D_{R}$, where each $D_{i}$ is a stable parabolic bundle of rank $n_{i}$, degree $-k_{i}$ and with weights $\gamma^{\sigma_{t}}=\left(\gamma_{\sigma_{1}(1)}, \ldots, \gamma_{\sigma_{l}\left(n_{t}\right)}\right)$. With the obvious definitions for $\sigma, \mathbf{k}$ and $\mathbf{n}$, this determines a $\gamma$-partition $\xi=(\sigma, \mathbf{k}, \mathbf{n})$. Furthermore, each of $D_{1}, \ldots, D_{R}$ is completely determined by $\xi$ as a topological parabolic bundle. For any $\xi \in \pi(\gamma)$, we define the stratum $\sum_{\zeta}^{\prime \prime}$ by

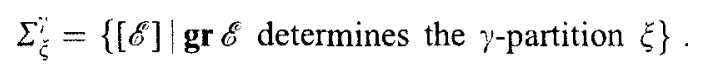

It is clear that $[\mathscr{E}]$ and $\left[\mathscr{E}^{\prime}\right]$ are in the same stratum if and only if each of the components $D_{i}$ and $D_{i}^{\prime}$ of $\mathbf{g r} \mathscr{E}$ and $\mathbf{g r} \mathscr{E}^{\prime}$ are isomorphic as topological parabolic bundles (up to reordering). Including the trivial partition in $\pi(\gamma)$, whose stratum is $\mathscr{M}^{\prime} \backslash \Sigma$, we have

$$
\mathscr{M}^{i}=\bigcup_{\zeta \in \pi(\xi)} \Sigma_{\zeta}^{\prime} .
$$

It is not difficult to identify $\Sigma_{\bar{\zeta}}^{\prime \prime}$ with the smooth part of a product of moduli of lower rank bundles. Since each $[\mathscr{E}] \in \Sigma_{\xi}^{\prime}$ has gr $\mathscr{E} \cong D_{1} \oplus \ldots \oplus D_{R}$ topologically, the holomorphic structure of $\mathbf{g r} \mathscr{E}$ determines a point in the product of moduli of lower rank bundles. More precisely, the assignment $[\mathscr{E}] \mapsto\left(\left[D_{1}\right], \ldots,\left[D_{R}\right]\right)$ defines an injective map $\sum_{\zeta}^{\ddot{\prime}} \rightarrow \mathscr{M}^{\sigma_{1}} \times \ldots \times \mathscr{M}^{\sigma_{R}}$, where $\gamma^{\sigma_{i}}$ denotes the weights inherited by $D_{l}$. Further, since each $D_{l}$ is stable, the image of this map is equal to the product of the smooth part of each moduli, and we have

$$
\Sigma_{\xi}^{i}=\prod_{i=1}^{R}\left(\mathscr{M}^{\sigma_{i}} \backslash \Sigma^{\sigma^{\sigma_{i}}}\right)
$$

where $\sum^{i^{\sigma_{1}}}$ refers to the subset of $\mathscr{M}^{\sigma_{l}}$ that comes from strictly semistable bundles. (Incidentally, if $\gamma \in \hat{\partial}_{e} W$, and $D_{i}$ and $D_{t^{\prime}}$ are isomorphic as topological parabolic bundles, then one needs to replace the direct product above with an obviously defined symmetric product.) The closure of a stratum is given by

$$
\overline{\sum_{\bar{\zeta}}^{i}}=\prod_{i=1}^{R} \mathscr{M}^{\sigma_{i}} .
$$

We can describe this more effectively by defining a partial ordering on the set $\pi(\gamma)$ of partitions by refinement, i.e. if $\xi, \xi^{\prime} \in \pi(\gamma)$ then we write $\xi^{\prime} \prec \xi$ if $\xi^{\prime}$ is a proper refinement of $\xi$. It should now be clear that

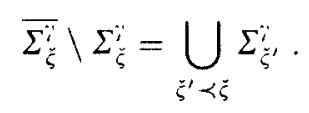

We say that $\xi \in \pi(\gamma)$ is minimal if there is no $\xi^{\prime} \in \pi(\gamma)$ with $\xi^{\prime} \prec \xi$. So, whenever $\xi$ is minimal the stratum $\Sigma_{\zeta}^{\prime \prime}$ is closed. Finally, notice that the closure of two strata have nonempty intersection if and only if their defining 
partitions have a common refinement in $\pi(\gamma)$. This is a consequence of the general intersection formula

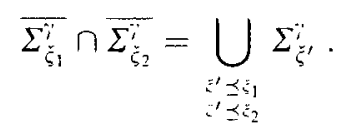

Definition 4.4. An algebraic map $f: X \rightarrow Y$ is called weakly stratified with respect to the stratifications $X=\bigcup_{\alpha} X_{x}$ and $Y=\bigcup_{\beta} Y_{\beta}$ if $f^{-1}\left(Y_{\beta}\right) \subset X_{\alpha}$ and if $f$ restricted to $f^{-1}\left(Y_{\beta}\right)$ is a fibration.

Theorem 4.5. If $\beta, \gamma \in \stackrel{\circ}{W}$ and $\beta>\gamma$, then the map $\phi_{\beta}: \mathscr{M}^{\beta} \rightarrow \mathscr{M}$ is weakly stratified with respect to the natural stratifications on $\mathscr{A}^{\beta}$ and $\mathscr{M}$. Moreover, $\phi_{\beta}$ restricted to the preimage of any stratum is a fibration tower whose fibers are all projective spaces.

Proof. For two elements $a, b$ in a poset $P$, we say that $a$ covers $b$ if $a>b$ and there is no $c$ with $a>c>b$. We will use this terminology for both the poset of weights and the poset $\pi(\gamma)$ of $\gamma$-partitions.

Choosing a maximal chain of weights

$$
\beta=\beta_{0}>\beta_{1}>\ldots>\beta_{m}=\gamma,
$$

we see that the map $\phi_{\beta}$ is the composite of the following sequence of maps

$$
\mathscr{M}^{\beta}=\mathscr{A}^{\beta_{0}} \rightarrow \mathscr{M}^{\beta_{1}} \rightarrow \ldots \rightarrow \mathscr{M}^{\beta_{n}}=\mathscr{M}^{i},
$$

where each $\beta_{i}$ covers $\beta_{i+1}$. Thus, it suffices to prove the following claim.

Claim. If $\beta$ covers $\gamma$, then for any stratum $\Sigma_{\eta}^{i}$, there is a stratum $\Sigma_{\xi}^{\beta}$ with $\phi_{\beta}^{-1}\left(\Sigma_{\eta \eta}^{i}\right) \subset \Sigma_{\check{\zeta}}^{\beta}$ so that the restriction of the map $\phi_{\beta}: \mathscr{M}^{\beta} \rightarrow \mathscr{M}^{\prime}$ to $\phi_{\beta}^{-1}\left(\Sigma_{\eta \eta}^{i}\right)$ is a projective bundle.

The theorem follows from this by induction on the length of the chain.

Before embarking on the proof of the claim, we make some general remarks about the geometric significance of the posets involved. First, notice that $\beta>\gamma$ implies that $\pi(\beta) \subset \pi(\gamma)$. Geometrically this means that any hyperplane containing $\beta$ also contains $\gamma$. Let $\left\{H_{1}, \ldots, H_{m}\right\}$ be the set of hyperplanes containing $\beta$ and $\left\{H_{1}, \ldots, H_{M}\right\}$ be those containing $\gamma$. Clearly $\beta>\gamma \Rightarrow M>m$. Suppose $\lambda$ is a weight with $\beta>\lambda>\gamma$, then by reordering, we see that $\lambda \notin H_{k}$ for some $m<k \leqq M$. Conversely, if $\beta$ covers $\gamma$ then each of the hyperplanes $H_{m+1}, \ldots, H_{M}$ is essential in the sense that for any $m<k \leqq M$, we have

$$
\left(\bigcap_{i=1}^{m} H_{i}\right) \cap H_{k}=\bigcap_{i=1}^{M} H_{i} .
$$

Thus, if $M>m+1$, we see that the hyperplanes $\left\{H_{1}, \ldots, H_{M}\right\}$ cannot be in general position. 
Assume that $\beta$ covers $\gamma$. Because the map $\phi_{\beta}: \mathscr{M}^{\beta} \rightarrow \mathscr{M}^{i}$ is surjective, for any $[\mathscr{F}] \in \mathscr{M}$, we have some $[\mathscr{E}] \in \mathscr{M}^{\beta}$ with $\phi_{\beta}([\mathscr{E}])=[\mathscr{F}]$. But the inclusion $\mathscr{C}_{s s}^{\beta} \subset \mathscr{C}_{s s}^{\prime}$ of Proposition 4.1 shows that $\mathscr{E} \in \mathscr{C}_{s s}^{\prime}$, and so $\mathbf{g r}_{\mathscr{E}} \mathscr{E} \cong \mathbf{g r}_{.} \mathscr{F}$ as holomorphic parabolic bundles. Moreover, letting $\eta \in \pi(\gamma)$ and $\xi \in \pi(\beta)$ denote the partitions induced by $\mathbf{g r}_{;} \mathscr{F}$ and $\mathbf{g r}_{\beta} \mathscr{E}$, respectively, we see that $\eta$ is a (not necessarily proper) refinement of $\xi$, i.e. $\eta \preceq \xi$ in the set $\pi(\gamma)$ (recall that $\pi(\beta) \subset \pi(\gamma)$ ). Since $\beta$ covers $\gamma$, it follows that the lengths of $\eta$ and $\xi$ are related by $|\xi| \leqq|\eta| \leqq|\xi|+1$ (otherwise, if $|\eta|>|\xi|+1$, then this would contradict the condition (8)). Thus we have two cases: either $|\eta|=|\xi|$, which implies $\eta=\xi$, or $|\eta|=|\xi|+1$ and it follows that $\xi$ covers $\eta$. (Note that $\xi$ covers $\eta$ if and only if $\eta \prec \xi$ and $|\eta|=|\xi|+1$.) Summarizing, we have

Lemma 4.6. Suppose that $\beta$ covers $\gamma$ and $\eta \in \pi(\gamma)$. Then either $\eta \in \pi(\beta)$, or there is a unique $\xi \in \pi(\beta)$ so that $\xi$ covers $\eta$ in the set $\pi(\gamma)$.

Proof.. The only thing left to show is uniqueness, so suppose $\eta \notin \pi(\beta)$ and suppose that $\xi_{1}, \xi_{2} \in \pi(\beta)$ both cover $\eta$. Writing $\xi_{i}=\left(\sigma^{i}, \mathbf{k}^{i}, \mathbf{n}^{l}\right)$ and $\eta=$ ( $\tau, \mathbf{h}, \mathbf{m})$, since $\eta \notin \pi(\beta)$, we have exactly two elements of $\tau=\left(\tau_{1}, \ldots, \tau_{R+1}\right)$, say $\tau_{R}$ and $\tau_{R+1}$, which are distinguished by the fact that $\sum_{j=1}^{m_{R}} \beta_{\tau_{R}(j)} \notin \mathbf{Z}$ and $\sum_{j=1}^{m_{R+1}} \beta_{\tau_{R, 1}(j)} \notin \mathbf{Z}$. But then a straightforward argument shows that up to reordering, $\sigma_{i}^{1}=\tau_{i}=\sigma_{1}^{2}$ for $1 \leqq i<R$ and $\sigma_{R}^{1}=\tau_{R} \cup \tau_{R+1}=\sigma_{R}^{2}$. Now it follows that $\xi_{1}=\xi_{2}$.

We can now conclude the proof Theorem 4.5.

proof of claim. First, we use the above lemma to identify the appropriate stratum $\Sigma_{\xi}^{\beta}$ which contains $\phi_{\beta}^{-1}\left(\Sigma_{\eta}^{\prime \prime}\right)$. On the one hand, if $\eta \in \pi(\beta)$, then using $\Sigma_{\eta}^{\beta}$, we see that the map $\phi_{\beta}$ restricted to the preimage of $\Sigma_{\eta}^{\prime \prime}$ is an isomorphism. Otherwise if $\eta \notin \pi(\beta)$, pick the unique $\xi \in \pi(\beta)$ which covers $\eta$. As in the proof of Lemma 4.6 , let $\xi=(\sigma, \mathbf{k}, \mathbf{n})$ and $\eta=(\tau, \mathbf{h}, \mathbf{m})$ where $\sigma_{l}=\tau_{t}$ for $1 \leqq i<R$ and $\sigma_{R}=\tau_{R} \cup \tau_{R+1}$. Thus

$$
\begin{aligned}
& \Sigma_{\zeta}^{\beta}=\prod_{i=1}^{R}\left(\mathscr{M}^{\beta^{\sigma_{i}}} \backslash \Sigma^{\beta^{\sigma_{i}}}\right), \\
& \Sigma_{\eta}^{i}=\prod_{i=1}^{R+1}\left(\mathscr{M}^{\tau_{i}} \backslash \Sigma^{i^{\tau_{i}}}\right) .
\end{aligned}
$$

Choose a path $\beta_{t}$ connecting $\beta$ to $\gamma$ so that for all $t \in[0,1)$, the weight $\beta_{t}$ lies in the same chamber as $\beta$. It is helpful to use the partition $\sigma$ to think of the weights as points on the product of weight spaces for lower rank bundles, i.e. writing $\beta^{\sigma_{t}}=\left(\beta_{\sigma_{t}(1)}, \ldots, \beta_{\sigma_{t}\left(n_{t}\right)}\right)$, for $1 \leqq i \leqq R$, then $\beta_{t}$ can be viewed as a path on $W_{1} \times \ldots \times W_{R}$, where $W_{i}$ is the weight space for the moduli $\mathscr{M}^{\beta^{\sigma_{i}}}$. Viewing each $\beta_{t}^{\sigma_{t}}$ as a path on $W_{i}$, we see that because $\xi$ covers $\eta$, for $t \in[0,1]$ and $1 \leqq i<R, \beta_{t}^{\sigma_{t}}$ lies in the same chamber as $\beta^{\sigma_{1}}$. Now Lemma 2.7 shows that $\mathscr{M}^{\beta^{a_{i}}}$ and $\mathscr{M}^{i_{1}}$ define identical moduli for $1 \leqq i<R$. So we just need to analyze the map $\mathscr{M}^{\beta^{\sigma_{R}}} \longrightarrow \mathscr{M}^{\sigma_{R}}$. For $i=R$, we see that $\beta_{t}^{\sigma_{R}}$ lies in the same chamber as $\beta^{\sigma_{R}}$ for $t \in[0,1)$, but that $\beta_{1}^{\sigma_{R}}=\gamma^{\sigma_{R}}$ encounters 
some new hyperplanes in $W_{R}$, i.e. as weights, $\beta^{\sigma_{R}}$ covers $\gamma^{\sigma_{R}}$. The fact that $\left(\tau_{R}, \tau_{R+1}\right)$ is a length 2 partition of $\sigma_{R}$ shows that

$$
\left(\mathscr{M}^{i^{\tau_{R}}} \backslash \Sigma^{i^{T_{R}}}\right) \times\left(\mathscr{M}^{i^{\tau_{R} 11}} \backslash \sum^{i^{\top} R+1}\right)
$$

is the smooth part of the singular locus in $\mathscr{M}^{{ }^{\circ}}{ }$ and thus we can apply the proof of Theorem 3.1 to see that the restriction of the map $\mathscr{M}^{\beta^{\boldsymbol{a}_{R}}} \rightarrow \mathscr{A}^{a^{a_{R}}}$ to

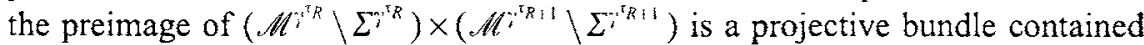

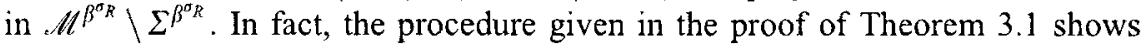
how to determine the projective space fiber. This completes the proof of the claim and also finishes the theorem.

Theorem 4.5 is the extension of the variation theorem to very singular moduli spaces. There is also an extension of the degeneration theorem in this case which we now describe. Suppose $\gamma \in \stackrel{\circ}{W}$ and $\delta \in \partial_{e} W$ are in the same chamber. Then, up to the choices given by the fact that the weights of $\delta$ are not distinct, it follows that $\alpha$ and $\delta$ have identical partition sets, i.e. $\pi(\gamma)=$ $\pi(\delta)=\pi$ and we see that

$$
\mathscr{M}^{i}=\bigcup_{\zeta \in \pi} \Sigma_{\zeta}^{i} \text { and } \mathscr{M}^{\delta}=\bigcup_{\zeta \in \pi} \Sigma_{\zeta}^{\delta} .
$$

Notice that the canonical map $\phi_{i}: \mathscr{M}^{i} \rightarrow \mathscr{M}^{\delta}$ is a weakly stratified map, i.e. $\phi_{i}\left(\Sigma_{\xi}^{\ddot{z}}\right)=\Sigma_{\zeta}^{\delta}$. Identifying the strata with products of moduli of parabolic bundles of lower rank (here, by product we mean symmetric product when appropriate), Proposition 3.4 applies one stratum at a time to prove the following.

Proposition 4.7. The natural projection $\mathscr{M}^{i} \rightarrow \mathscr{M}^{\delta}$ is a weakly stratified map whose fiber over any stratum can be determined from Proposition 3.4.

Before closing this section, we illustrate one interesting phenomenon. Suppose $\gamma$ is a weight admitting a length $R$ partition $\xi$. Then an elementary counting argument shows that $\gamma$ admits at least

$$
\frac{1}{2} \sum_{k=1}^{R-1}\left(\begin{array}{l}
R \\
k
\end{array}\right)=2^{R-1}-1
$$

distinct length 2 partitions. Thus, if $\left\{H_{1}, \ldots, H_{m}\right\}$ denotes the set of all hyperplanes containing $\gamma$, then we must have $m \geqq 2^{R-1}-1$. Furthermore, if $R>2$, then these hyperplanes are not in general position. This follows simply by considering the special case where $\gamma$ admits a length 3 partition. Then there are 3 hyperplanes $\left\{H_{1}, H_{2}, H_{3}\right\}$ containing $\gamma$ with the property that the intersection of any two is equal to the intersection of all three. Consequently, these hyperplanes are not in general position.

A simple example of such a weight is given by $\gamma=\left(\frac{1}{10}, \frac{1}{5}, \frac{2}{5}, \frac{3}{5}, \frac{4}{5}, \frac{9}{10}\right)$. Notice that the set of $\gamma$-partitions is given by

$$
\pi(\gamma)=\left\{\xi_{1}, \xi_{2}, \xi_{3}, \eta\right\}
$$



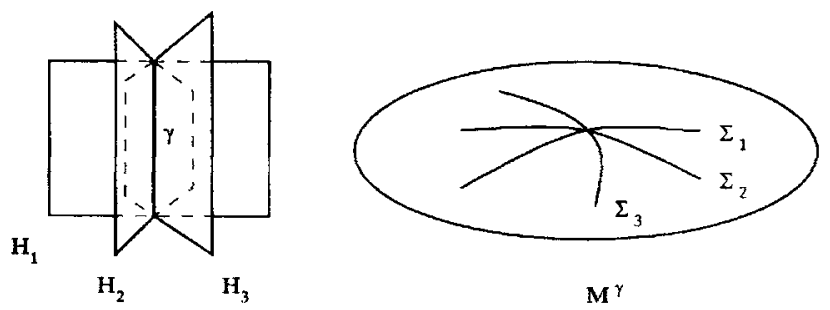

Fig. 1. The singular locus of $\mathscr{N}$ i when the hyperplanes containing $\gamma$ are not in general position.
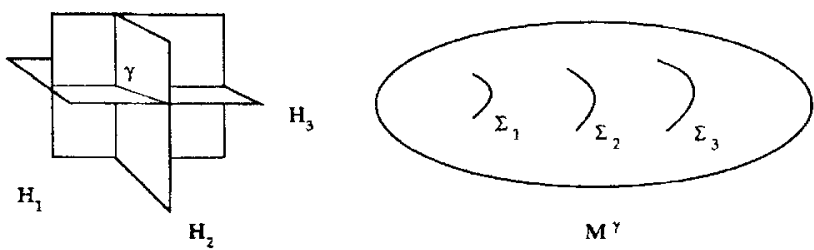

Fig. 2. The singular locus of $\mathscr{M}$ in whe hyperplanes containing $\gamma$ are in general position.

where each $\xi_{i}$ has length two and corresponds to a hyperplane $H_{i}$, and $\eta$ is a length three partition which is a refinement of each $\xi_{i}$. Thus we see by the intersection formula (7) that the closures of the strata corresponding to the $\xi_{l}$ intersect nontrivially, i.e. $\bigcap_{i=1}^{3} \overline{\Sigma_{\xi_{1}}^{\gamma}}=\Sigma_{\eta}^{i}$ (see Fig.1).

On the other hand, suppose that the set $\left\{H_{1}, \ldots, H_{m}\right\}$ of hyperplanes containing $\gamma$ are in general position. Then the above considerations show that $\gamma$ does not admit any partitions of length $>2$. Consequently, the intersection formula (7) shows that each stratum is closed. For a concrete example, consider $\gamma=\left(\frac{1}{20}, \frac{1}{10}, \frac{1}{5}, \frac{1}{4}, \frac{3}{10}, \frac{2}{5}, \frac{7}{10}\right)$. Then $\pi(\gamma)=\left\{\xi_{1}, \xi_{2}, \xi_{3}\right\}$ consists entirely of length two partitions, and the resulting moduli is pictured in Fig. 2.

We close this section with the following conjecture.

Conjecture 4.8. For any $\gamma \in \stackrel{\circ}{W}$, there exists generic $\alpha>\gamma$ so that the canonical map $\phi_{x}: \mathscr{M}^{\alpha} \rightarrow \mathscr{M}^{i}$ is a small resolution.

The proof given in [17] applies in the case when the hyperplanes $\left\{H_{1}, \ldots, H_{m}\right\}$ containing $\gamma$ are in general position, but this is rare by the preceding discussion.

\section{Multiple parabolic points}

We now consider the case of smooth curves of arbitrary genus $g$ and with $m$ distinct marked points $\left\{p_{1}, \ldots, p_{m}\right\}$. Then a topological parabolic bundle over $X$ is by definition a $\mathbf{C}^{n}$ bundle $E$ with weighted flags over each $p_{j}$. The other definitions, namely the parabolic slope, stability, the weight space, etc., all 
extend to this situation in a more-or-less obvious way. For a detailed account of this and the construction of the moduli in this case the reader is referred to [24]. The moduli is a normal projective variety and is smooth for a choice of generic weights. Its dimension is given by

$$
\operatorname{dim} \mathscr{M}^{\alpha}=(g-1) n^{2}+1+\sum_{j=1}^{m} \operatorname{dim} \mathscr{F}_{p_{j}},
$$

where $\mathscr{F}_{p}$, is the flag variety describing the quasi-parabolic structure at $p_{j}$. In Sect. 2.2, we defined the weight space as consisting of those weights $\alpha$ with $\mu_{x}(E)=0$. However, this was purely a matter of convenience and in this section, we allow for general weights. Notice that the collection of weights so that $\mathscr{C}_{s S S}^{x} \neq \varnothing$ still consists of a union of hyperplanes determined by the equation $\mu_{x}\left(E^{\prime}\right)=\mu_{x}(E)$ for a subbundle $E^{\prime}$. To deal with the anomalies in low genus, we shall call a weight $\alpha$ sufficiently generic if $C_{s}^{\alpha} \neq \varnothing$. The proofs of Theorem 3.1 and Proposition 3.4 transfer to this situation to give identical results, provided the weights involved are sufficiently generic.

Furthermore, these results also extend to the fixed determinant moduli as we now explain. Let $J(k)$ denote the Jacobian of line bundles over $X$ of degree $k$ and for $\Lambda \in J(k)$, consider $\mathscr{C}_{s s}^{x, 0}=\left\{\mathscr{E}^{\mathscr{E}} \in \mathscr{C}_{s s}^{x} \mid \operatorname{det} \mathscr{E}=\Lambda\right\}$ with moduli $\mathscr{V}^{x}$ defined to be $\mathscr{C}_{s s}^{x, 0}$ modulo $s$-equivalence. We call $\mathscr{N}^{x}$ the moduli of bundles with fixed determinant. The fibration

$$
\mathscr{N}^{\alpha} \rightarrow \mathscr{M}^{\alpha} \stackrel{\text { det }}{\rightarrow} J(k)
$$

shows that for sufficiently generic weights

$$
\operatorname{dim} \mathscr{N}^{\alpha}=(g-1)\left(n^{2}-1\right)+\sum_{j=1}^{m} \operatorname{dim} \widetilde{\mathscr{F}}_{p_{j}} .
$$

By applying identical arguments as in the proof of Theorem 3.1 to bundles with fixed determinant, one can prove that $\mathscr{N}^{\alpha}$ and $\mathscr{N}^{\beta}$ are related by a special birational transformation whenever $\alpha$ and $\beta$ are generic, sufficiently generic weights on either side of a hyperplane. However, it should be mentioned that the moduli of strictly semistable bundles, which we continue to denote by $\Sigma ;$, is no longer a product of moduli of lower dimension (except when $g=0$ ).

We now proceed with a discussion of rationality of the moduli spaces of parabolic bundles in genus zero. One of the special features in this case is that for certain weights, the moduli space is empty. One way to see this for "traceless" weights $\alpha$ satisfying pardeg ${ }_{x} E=0$ is to relate the moduli to certain representation spaces, which can be shown to be empty for some choice of weights. However, we give a direct argument utilizing Grothedieck's theorem [13] that any bundle $E$ of rank $n$ and degree $k$ over $\mathbf{P}^{1}$ is the direct sum of line bundles. We remind the reader that we no longer make the assumption that $\operatorname{pardeg}_{x} E=0$.

Consider $\mathbf{P}^{1}$ with marked points $\left\{p_{1}, \ldots, p_{m}\right\}$ and suppose that $E$ is a quasiparabolic bundle of rank $n>1$ and degree $k$ and with full flags over each of the parabolic points. We can assume, by tensoring with a line bundle if necessary, that $0 \leqq k<n$. Consider the two cases: $0<k<n$ and $k=0$. 
If $0<k<n$, then choose generic small weights, specifically, $0<x_{1}^{j}<$ $\ldots<\alpha_{n}^{\prime}<\frac{1}{n^{2} m}$ over $p_{j}$. Then $\operatorname{pardeg}_{\alpha} E<k+1 / n<n$ so $\mu_{x}(E)<1$. If $E=L_{1} \oplus \ldots \oplus L_{n}$ is $\alpha$-semistable, writing $k_{i}=\operatorname{deg} L_{i}$, then $k_{i}+\sum_{j} \alpha_{1}^{j} \leqq$ $\mu_{\alpha}\left(L_{i}\right) \leqq \mu_{\alpha}(E)<1$, which implies that $k_{\imath} \leqq 0$ and so $k=\sum k_{\imath} \leqq 0$, a contradiction.

If $k=0$ then choose generic weights so that $\alpha_{t}^{j}<\frac{1}{n^{3} m}$ for all $i, j$ except for $\alpha_{n}^{1}$, which satisfies $(1-1 / n)<\alpha_{n}^{1}<\left(1-1 / n^{2}\right)$ (recall $\left.n>1\right)$. Then $\mu_{x}(E)<$ $1 / n$ and by the same argument as in the previous case, if $E=L_{1} \oplus \ldots \oplus L_{n}$ is $\alpha$-semistable, then $\operatorname{deg} L_{l}=0$. By choosing nowhere zero sections $s_{t} \in \mathrm{H}^{0}\left(L_{t}\right)$, we see easily that $E$ is holomorphically trivial. It follows that there is a trivial line subbundle $L$ so that $L_{p_{1}}$ coincides with $F_{n}^{1}$ and therefore inherits the large weight $\alpha_{n}^{1}$. Thus $\mu_{x}(L)>\alpha_{n}^{1}>(1-1 / n) \geqq 1 / n>\mu_{x}(E)$, so again we see that $E$ is unstable.

Now let $\alpha$ and $\beta$ be generic weights with $\mathscr{N}^{\alpha} \neq \varnothing$ and $\mathscr{V}^{\beta}=\varnothing$. Obviously, we can choose $\alpha$ and $\beta$ on either side of a hyperplane. Let $\delta$ be a point on this hyperplane but not on any other hyperplane.

We claim that $\mathscr{N}^{\delta}$ is a product of two moduli of lower rank. To see this, notice that $\delta$ lies in exactly one hyperplane and the moduli has only strictly semistable bundles. This follows from Lemma 3.5 applied to this special case. Indeed, $\varnothing \neq \mathscr{C}_{s s}^{x} \subset \mathscr{C}_{s s}^{\delta}$ and $\mathscr{C}_{s}^{\delta} \subset \mathscr{C}_{s}^{\beta}=\varnothing$. Thus each $\mathscr{E} \in \mathscr{C}_{s s}^{\delta}$ is $s$-equivalent to a bundle of the form $\mathscr{E}^{\prime} \oplus \mathscr{E}^{\prime \prime}$, where $\mathscr{E}^{\prime}$ and $\mathscr{E}^{\prime \prime}$ are stable parabolic bundles of ranks $n^{\prime}$ and $n^{\prime \prime}$ and with weights $\delta^{\prime}$ and $\delta^{\prime \prime}$, respectively. This implies that $\mathcal{N}^{\delta}=\Sigma^{\delta}=\mathcal{N}^{\delta^{\prime}} \times \mathcal{N}^{\delta^{\prime \prime}}$ for the partition $\left(\delta^{\prime}, \delta^{\prime \prime}\right)$ of $\delta$.

By induction, each $\mathscr{N}^{\delta^{\prime}}$ and $\mathscr{N}^{\delta^{\prime \prime}}$ is rational, (the case of rank 1 being trivial, while rank 2 is covered in [2]).

Indeed, we know more than this from the fact that $\mathscr{N}^{\beta}=\varnothing$. Assume (wlog) that $\mu_{x}\left(\mathscr{E}^{\prime}\right)<\mu_{\delta}\left(\mathscr{E}^{\prime}\right)<\mu_{\beta}\left(\mathscr{E}^{\prime}\right)$. Then since $\mathscr{N}^{\beta}=\varnothing$, by the results in Sect. 3.2, there are no nontrivial extensions of $\mathscr{E}^{\prime \prime}$ by $\mathscr{E}$, when regarded with weight $\beta$, i.e. $\operatorname{ParExt}{ }_{\beta}^{\prime}\left(\mathscr{E}^{\prime \prime \prime}, \mathscr{E}^{\prime}\right)=0$. In particular, our algorithm for computing $e_{\beta}$ gives $e_{\beta}=-1$, so that $e_{\alpha}=\operatorname{dim} \mathscr{M}^{\alpha}-\operatorname{dim} \Sigma^{\delta}$. (The previous formulation of this in terms of codimensions no longer makes sense.) It now follows easily that $\mathscr{N}^{\alpha} \rightarrow \mathscr{N}^{\delta}$ is a fiber bundle whose typical fiber is $\mathbf{P}^{e_{x}}=\operatorname{Proj}\left(\mathrm{H}^{1}\left(\operatorname{Parhomt}_{x}\left(\mathscr{E}^{\prime \prime \prime}, \mathscr{E}^{\prime \prime}\right)\right)\right)$. Hence the rationality of $\mathcal{H}^{-x}$ for any $\alpha$ with nontrivial moduli now follows.

Proposition 5.1. If the genus of $X$ is 0 , then for every $\alpha \in W, \mathcal{N}^{x}$ is rational provided it is nonempty. As a consequence, such moduli are simply connected.

To close this paper, we would like to emphasize the possibility of comparing, in the spirit of Theorem 3.1, the moduli spaces of parabolic bundles whose underlying vector bundles have different degrees. (One might think of this as a generalization of Hecke correspondence in the parabolic setting.)

Acknowledgement. We would like to thank Igor Dolgachev and Gordana Matic for several helpful discussions, in addition to the mathematics community of Michigan and Michigan State for supporting and encouraging this work. We are also grateful to the referee for making 
several corrections and suggestions which improved this paper. The first author was partially supported by a Rackham grant from the University of Michigan.

\section{References}

1. M. Atiyah and R. Bott, The Yang-Mills equations on a Riemann surface, Phil. Trans. Roy. Soc. Lond. A., 308 (1982), 524-615.

2. S. Bauer, Parabolic bundles, elliptic surfaces and SU(2)-representation spaces of genus zero Fuchsian groups, Math. Ann., 290 (1991), 509-526.

3. A. Bertram and Q. Szenes, Hilbert Polynomials of moduli spaces of rank 2 vector bundles II, Topology, 32 (1993) 599-609.

4. H.U. Boden, Representations of orbifold groups and parabolic bundles, Comm. Math. Helv., 66 (1991), 389-447.

5. H.U. Boden, Unitary Representations of Brieskorn spheres, MPI preprint 1993, to appear in Duke Math. J.

6. S. Bradlow and G. Daskalopoulos, Moduli of stable pairs for holomorphic bundles over Riemann surfaces, Int. J. Math., 2 (1991) 477-513.

7. G. Daskalopoulos and R. Wentworth, Geometric quantization for the moduli space of vector bundles with parabolic structure, preprint, 1991, to appear in Duke Math. Journal.

8. I. Dolgachev and $\mathrm{Y}$. Hu, Variation of Geometric Invariant Theory Quotients, preprint (1994), alg-geom/9402008.

9. C. Frohman and A. Nicas, An intersection homology invariant for knots in a rational homology sphere, Topology, 33 (1994) 123-158.

10. W. Goldman, The symplectic nature of fundamental groups of surfaces, Adv. Math., 54 (1984), 200-225.

11. R.M. Goresky, R.D. MacPherson, On the topology of algebraic torus actions, Lecture Notes in Math. 1271, 73-90, Springer-Verlag 1986.

12. R.M. Goresky and R.D. MacPherson, Intersection Homology II, Inven. Math., 71 (1983), $77-129$.

13. A. Grothendieck, Sur la classification des fibrés holomorphes sur la sphère de Riemann, Amer. J. Math., 79 (1957), 121-138.

14. A. Grothendieck and J. Dieudonné, Éléments de géométrie algeébrique III, (Seconde Partie). Inst. Hautes Études Sci. Publ. Math 17 (1963).

15. V. Guillemin and S. Sternberg, Birational equivalence in symplectic category, Invent. Math., 97 (1989), 485-522.

16. F. Hirzebruch, Topological Methods in Algebraic Geometry, Springer-Verlag, New York 1978.

17. Y. Hu, The geometry and topology of quotient varictics of torus actions, Duke Math. Journal, 68 (1992), 151-184.

18. F. Kirwan, On the homology of compactifications of moduli spaces of vector bundles over a Riemann surface, Proc. Lond. Math. Soc., 53 (1986), 237--266.

19. V. Mehta and C. Seshadri, Moduli of vector bundles on smooth curves with parabolic structures, Math. Ann., 248 (1980), 205-239.

20. D. Mumford and J. Fogarty, Geometric Invariant Theory, A Series of Modern Surveys in Mathematics, Springer-Verlag (1982).

21. P. Newstead, Topological properties of some spaces of stable bundles, Topology, 6 (1967) 241-262.

22. P. Newstead, Rationality of moduli spaces of stable bundles, Math. Ann., 215 (1975) 251-268. Math. Ann., 249 (1980), 281-282.

23. N. Nitsure, Cohomology of the moduli of parabolic bundles, Proc. Ind. Math. Soc., 95 (1986), 61-77.

24. C. Seshadri, Fibrés vectoriels sur les courbes algébriques, Asterisque, 96 (1982).

25. S. Smale, The structure of manifolds, Amer. J. Math., 84 (1962), 387-399.

26. M. Thaddeus, Stable pairs, linear systems and the Verlinde formula, Inven. Math., 117 (1994), 317-353.

27. L. Tu, Semistable bundles over an elliptic curve, Adv. in Math., 98, 1-26 (1993) 